\title{
Remote Doping of Scalable Nanowire Branches
}

\author{
Martin Friedl, ${ }^{\#}$ Kris Cerveny," Chunyi Huang, Didem Dede, Mohammad Samani, Megan O. Hill, \\ Nicholas Morgan, Wonjong Kim, Lucas Güniat, Jaime Segura-Ruiz, Lincoln J. Lauhon, \\ Dominik M. Zumbühl, and Anna Fontcuberta i Morral*
}

Cite This: Nano Lett. 2020, 20, 3577-3584

Read Online

亗 Metrics \& More

Article Recommendations

Supporting Information

ABSTRACT: Selective-area epitaxy provides a path toward high crystal quality, scalable, complex nanowire networks. These highquality networks could be used in topological quantum computing as well as in ultrafast photodetection schemes. Control of the carrier density and mean free path in these devices is key for all of these applications. Factors that affect the mean free path include scattering by surfaces, donors, defects, and impurities. Here, we demonstrate how to reduce donor scattering in InGaAs nanowire networks by adopting a remote-doping strategy. Low-temperature magnetotransport measurements indicate weak anti-localization-a signature of strong spin-orbit interaction-across a nanowire $\mathrm{Y}$ -
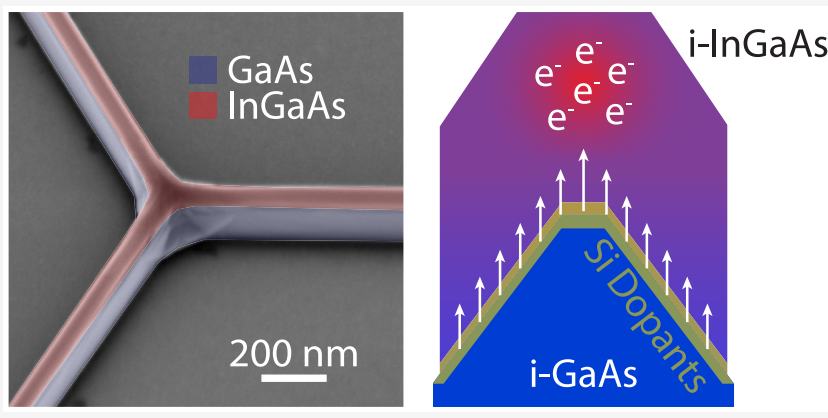
junction. This work serves as a blueprint for achieving remotely doped, ultraclean, and scalable nanowire networks for quantum technologies.

KEYWORDS: InGaAs, nanowires, selective-area epitaxy, weak anti-localization, spin-orbit interaction

\section{INTRODUCTION}

In the last two decades, semiconductor nanowires $(\mathrm{NWs})^{1}$ have opened multiple new perspectives in a wide variety of applications including photovoltaics, ${ }^{2-6}$ photodetectors, ${ }^{7-10}$ lasers, ${ }^{11-15}$ single-photon emitters, ${ }^{16,17}$ photoelectrochemistry, ${ }^{18}$ nanoscale electronics, ${ }^{19}$ and research into topological quantum computing. ${ }^{20-24}$ While most NW fabrication approaches result in free-standing structures, selective-area epitaxy (SAE) has recently been revived for the fabrication of horizontal NW assemblies that can be directly contacted on a wafer. $^{25,26} \mathrm{SAE}$ has thus been used to obtain lateral III-V NWs, with high NW crystal quality and with scalability unmatched by any other bottom-up NW growth method. The ability of SAE NWs to be grown into NW networks, ${ }^{27}$ and the relative ease with which they can be further processed directly on the original substrate, makes them extremely attractive for use in experiments exploring topological quantum transport physics. $^{28-32}$

It has generally been reported that surface scattering in NWs reduces carrier mean free paths which, by extension, also reduces carrier mobility with respect to their bulk counterparts. This is especially pertinent in InAs NWs, where the surface Fermi level pinning results in most of the conduction occurring at the surface. ${ }^{33}$ Room-temperature electrically measured mobilities for undoped, vertically grown InAs NWs thus fall in the range of $\sim 20-3000 \mathrm{~cm}^{2} /(\mathrm{V} \mathrm{s}){ }^{34-38}$ Noncontact measurement techniques such as $\mathrm{THz}$ pump-probe spectroscopy report slightly higher mobilities of $\sim 4000-6000 \mathrm{~cm}^{2} /$
(V s) due to the smaller length scale of the assessment and a possible selection bias (higher mobility sections contributing more to the signal). ${ }^{38-40}$ Still, mobilities remain significantly lower than the reported values for thin films of around 30000 $\mathrm{cm}^{2} /(\mathrm{V} \mathrm{s}) .^{41}$ InGaAs nanowires with In:Ga ratios around 50\%, on the other hand, have been reported with somewhat lower mobilities ranging from $\sim 500$ to $1500 \mathrm{~cm}^{2} /(\mathrm{V} \mathrm{s}){ }^{42,43}$

Over the years, modulation doping has proven to be a staple technique to many applications employing high-performance semiconductor heterostructures. This technique allows for the creation of two-dimensional electron gases (2DEGs) with high carrier concentration combined with extremely high mobilities. From groundbreaking scientific discoveries such as the integer and fractional quantum Hall effects, ${ }^{44-46}$ and enabling novel concepts such as topological qubits ${ }^{47-50}$ to high-power applications using high electron mobility transistors (HEMTs), ${ }^{51,52}$ a wide range of fields have benefited and are continuing to benefit from remotely doped 2DEG heterostructure schemes. With current knowledge and modern epitaxy techniques, state-of-the-art 2DEG devices can achieve mobilities regularly exceeding $10^{7} \mathrm{~cm}^{2} /(\mathrm{V} \mathrm{s}) .^{53,54}$ Similarly to

Received: February 7, 2020

Revised: April 9, 2020

Published: April 21, 2020 
bulk, direct doping of NWs reduces carrier mobilities due to ionized dopant scattering. To circumvent this carrier scattering mechanism, vertically grown free-standing GaAs NWs with a remote-doping design exhibit both high carrier densities and high mobility. ${ }^{55-58}$

In this work, we demonstrate the growth of quasi-1D InGaAs NW heterostructures hosting coherent transport by combining remote doping with SAE. The InGaAs NWs are obtained on a GaAs nanomembrane (NM) buffer in which the doping is located. The band alignment naturally results in the localization of electrons in the region with higher indium content (and lower bandgap). Improved electrical transport is demonstrated by low-temperature field-effect and magnetotransport measurements across InGaAs NW Y-branches, demonstrating high-quality NW junctions. This research thus lays the groundwork for future exploitation of remote-doping schemes in scalable NW networks, including materials beyond InGaAs.

\section{GROWTH}

Remotely doped InGaAs NWs were grown on top of GaAs $\mathrm{NM}$ buffers using a SAE approach, as depicted in Figure 1a. A
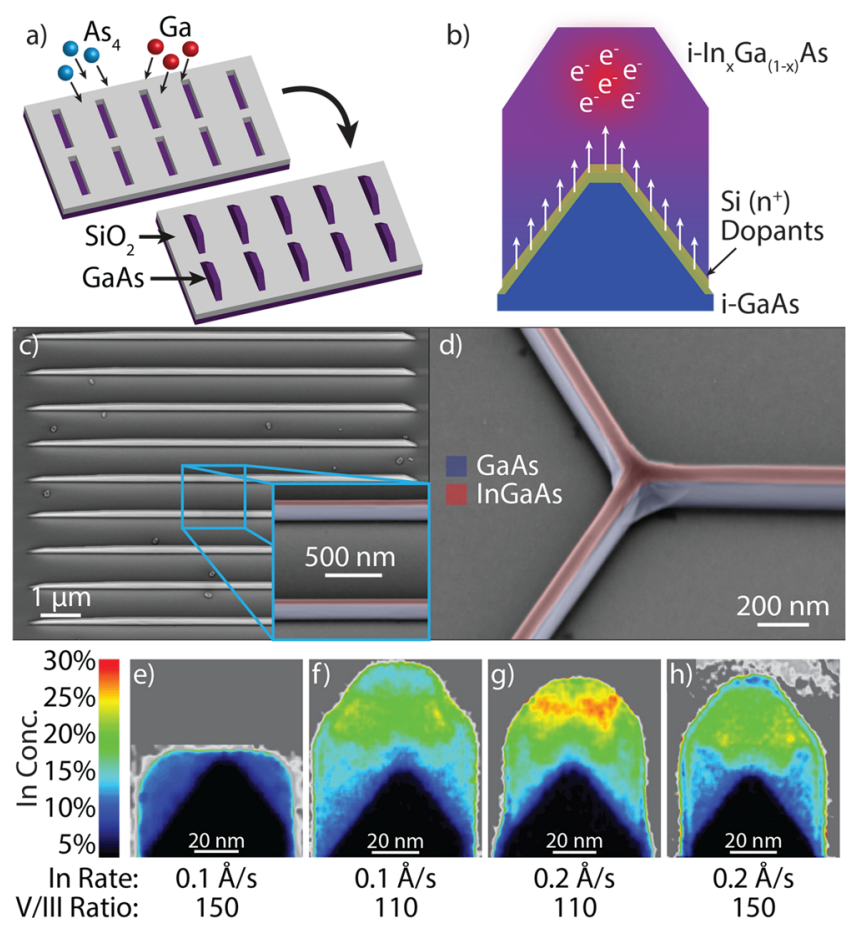

Figure 1. (a) Illustration of the SAE growth process. (b) Diagram showing the desired doping profile for remotely doped InGaAs NWs on GaAs NMs. (c) Tilted SEM image of an array of NWs after MBE growth. (d) Close-up tilted SEM image of a Y-junction showing uniform coalescence of InGaAs NWs. (e-h) Elemental maps of In concentration taken with STEM EDS on NW cross sections showing the dependence of growth conditions on NW composition.

GaAs ( $\left.\begin{array}{lll}1 & 1 & 1\end{array}\right) \mathrm{B}$ substrate was covered by a $\mathrm{SiO}_{2}$ mask to achieve high growth selectivity. The $\mathrm{SiO}_{2}$ mask was then patterned with stripes along the three equivalent $\left\langle\begin{array}{lll}1 & 1 & \overline{2}\end{array}\right\rangle$ directions on the substrate using e-beam lithography and reactive ion etching. The resist was stripped with an $\mathrm{O}_{2}$ plasma, and the substrate was etched in a dilute buffered HF solution before being loaded into the molecular beam epitaxy (MBE) cluster for growth to ensure an ultraclean surface.
Figure $1 \mathrm{~b}$ corresponds to the general scheme of the structures in this work. Silicon dopants are introduced at a distance from the InGaAs channel. We expect carriers to localize into the lower-bandgap NW region. As a result, the NWs benefit from an increased carrier concentration thanks to the extrinsic dopants, while ionized impurity scattering is limited due to the physical separation between the doped and transport regions.

Figure 1c shows a representative scanning electron microscopy (SEM) image of the remotely doped NW structures. We recognize a high degree of uniformity in both the buffer NMs and the NWs. Similarly, as shown in Figure 1d, Y-branched structures result from the merging of NWs growing in three equivalent directions. ${ }^{27}$ These branched structures exhibit a high degree of NW uniformity, which is further confirmed by X-ray fluorescence (XRF) measurements performed at the ID16B beamline of the European Synchrotron Radiation Facility (ESRF) shown in the Supporting Information.

In-Ga intermixing is commonly observed at the interface between InAs and GaAs. ${ }^{59}$ This is also the case for InAs grown on GaAs buffer NMs. In a previous study, we demonstrated InGaAs NWs with a relatively low In content. ${ }^{27}$ Increasing the In content is key to enhancing spin-orbit interaction (SOI). In this context, we explored different growth conditions to increase the In concentration. In particular, we varied temperature along with In and $\mathrm{As}_{4}$ fluxes. We present four representative samples with a variation in the In deposition rate and $\mathrm{As}_{4}$ beam equivalent pressure (BEP). The composition was analyzed by performing elemental maps by scanning transmission electron microscopy (STEM) energy dispersive X-ray spectroscopy (EDS) on prepared cross sections. The resulting In concentration maps are shown in Figure $1 \mathrm{e}-\mathrm{h}$. Here, the atomic concentration of In is calculated as $C_{\mathrm{In}} /\left(C_{\mathrm{In}}+C_{\mathrm{Ga}}+C_{\mathrm{As}}\right)$ with the maximum possible concentration being $50 \%$ for pure InAs. In Figure 1e, we see that an In rate of $0.1 \AA / \mathrm{s}$ and V/III ratio of 150 yields a selfterminating growth and a flat (111) top facet with an In concentration of $\sim 10 \%$ at the two upper corners of the structure. Decreasing the V/III ratio to 110, as shown in Figure $1 \mathrm{f}$, results in a more pointed structure that does not selfterminate. This results in a larger amount of material being deposited and a maximum In concentration of $\sim 20 \%$. Doubling the In deposition rate to $0.2 \AA / \mathrm{s}$ (while halving the growth time to keep the total deposited volume constant) gives a similarly shaped structure; however, now the peak In concentration is $\sim 25 \%$, as shown in Figure 1g. Finally, Figure $1 \mathrm{~h}$ shows the result of also increasing the V/III ratio to 150 with an In deposition rate of $0.2 \AA / \mathrm{s}$. We observe a structure very similar to Figure If with a pointed shape and maximum In concentration of $20 \%$.

We, therefore, observe that a higher In flux yields an increased In concentration in the NW, up to about $25 \%$. On the other hand, at low In rates, In concentrations of only $10 \%$ or less are observed. The InAs growth temperatures are relatively low for solid-phase diffusion which is typically only observed above $750{ }^{\circ} \mathrm{C} .^{60-62}$ The In-Ga intermixing could instead be mediated by surface adatom diffusion during growth. This is a thermodynamically driven effect which, consequently, can be suppressed at higher deposition rates where the system approaches a kinetic regime. ${ }^{63}$

Also, the V/III ratio is known to affect the atomic surface reconstruction which in turn affects facet-dependent growth 
rates and the resulting shape. ${ }^{64,65}$ In Figure 1e, at high V/III ratios and low growth rates, InGaAs grows to form a flat (111) B top facet, after which NW growth stops. This suggests that desorption of In species from this flat top facet is higher than the incoming flux. However, if the In rate is increased, as in Figure $1 \mathrm{~h}$, then NW growth is re-established which can be explained by the fact that the increased incoming flux becomes greater than the desorbing flux, thus continuing NW growth. Similarly, if the V/III ratio is decreased, as in Figure 1f, a similar effect is achieved. This, therefore, suggests that, at the higher V/III ratio, the abundance of As atoms also plays a role in inhibiting growth on the (111)B top facet. Thus, higher In rates and lower V/III ratios should be pursued to reduce Ga intermixing in the NW. This result is at least partially supported by recent reports on (100) GaAs substrates where very low $\mathrm{V} / \mathrm{III}$ ratios are used to grow pure InAs NWs on top of $\mathrm{GaAs}(\mathrm{Sb})$ buffers. $^{28}$

The highest In concentration was about $25 \%$ absolute, corresponding to $\mathrm{In}_{0.5} \mathrm{Ga}_{0.5}$ As. This sample was obtained with a high In flux and lower V/III ratio. These conditions were kept for the rest of the structures presented in this study.

\section{ATOM-PROBE TOMOGRAPHY}

As the silicon dopant concentration was well below the detection limits of STEM EDS, atom probe tomography (APT) was used as a technique to image the three-dimensional distribution of the dopants in the structure.

APT was used to analyze the distribution of the dopants in the nanowire heterostructures, as shown in Figure 2.27,66 Samples were prepared by lift-out and annular milling using a focused ion beam to isolate the nanowire region for analysis. $^{67,68}$ Details of the specimen preparation and APT analysis conditions can be found in the Experimental Section. Figure 2a shows the APT reconstruction of the region indicated by the dashed white line in the schematic. Silicon dopants were mostly detected on the GaAs top facet, as shown in Figure S8. The analysis here focuses on the subregion indicated in the dashed black line. To accurately measure the $\mathrm{Si}$ dopant distribution despite this artifact, a proximity histogram $^{69}$ was generated, as shown in Figure $2 b$ on either side of a $16 \%$ In mole fraction isosurface. The Si concentration peaks below the NM-NW interface, as expected from the doping scheme. APT analysis of two additional heterostructures (see the Supporting Information) found a similar distribution of $\mathrm{Si}$ concentrated at the NM-NW boundary. Moving 5-10 nm away from the interface, Si was not detected above the noise level (see Figure S8). The upper bounds on the $\mathrm{Si}$ concentration in the upper and lower regions away from the interface are $2 \times 10^{18}$ and $7 \times 10^{18} \mathrm{~cm}^{-3}$, respectively, as indicated by dashed lines in Figure $2 \mathrm{~b}$. These measurements demonstrate that $\mathrm{Si}$ was incorporated at a distance from the InGaAs transport channel, validating the remote doping from the compositional mapping point of view.

\section{MAGNETOTRANSPORT}

NW structures were electrically contacted for four-point measurements on all three arms of the Y-junctions (see the Experimental Section). A top gate was fabricated by first covering the junction with a $40 \mathrm{~nm}$ thick $\mathrm{HfO}_{2}$ gate oxide by atomic layer deposition (ALD) followed by metal evaporation. An SEM image of an electrical device illustrating the overall design is displayed in Figure 3a. A false-colored cross-sectional a)

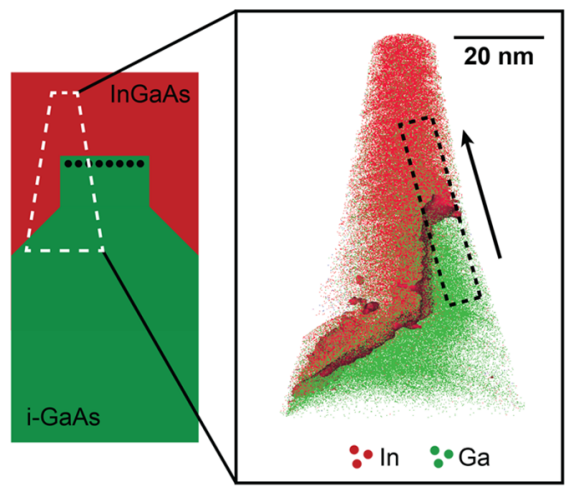

b)

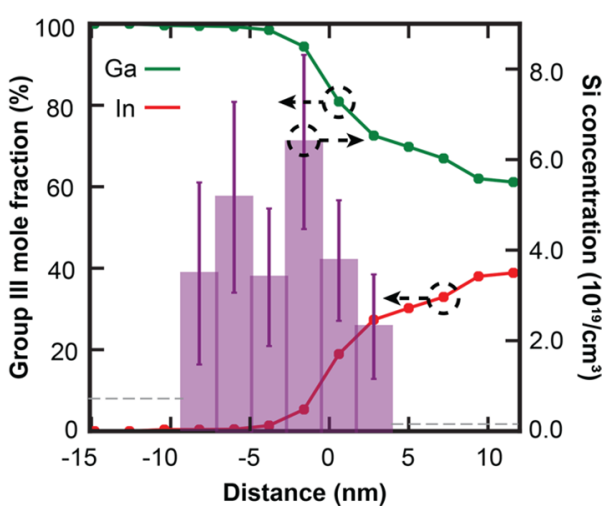

Figure 2. (a) APT reconstruction of an InGaAs NW on a GaAs NM. The white dashed line in the schematic indicates the region analyzed by APT. Ga and In atoms are shown in green and red, respectively. As atoms are omitted for clarity. The $\mathrm{NM}-\mathrm{NW}$ boundary is rendered with an isoconcentration surface of $5 \%$ In mole fraction. (b) Proximity histogram across the NM-NW interface showing the $\mathrm{Si}$ dopant distribution. Gray dashed lines show the upper bounds on $\mathrm{Si}$ concentration in regions away from the interface, where $\mathrm{Si}$ counts were not above the noise level. The isoconcentration surface of $16 \%$ In mole fraction is at $0 \mathrm{~nm}$ as a reference.

focused ion beam (FIB) STEM image is displayed in Figure $3 \mathrm{~b}$ with the identification of the different layers of the device. It is important to note that the metallic contact layers are continuous through the whole high-aspect-ratio structure, as a result of the dual-angle evaporation.

The samples were then cooled down and electrically characterized at cryogenic temperature. The conductance as a function of top gate voltage $V_{\mathrm{g}}$ is shown in Figure $3 \mathrm{c}$ in units of $e^{2} / h$, giving very similar results over all pairs of arms of the Y-junctions. From this measurement, the field-effect mobility is extracted using ${ }^{70}$

$$
G\left(V_{\mathrm{g}}\right)=\left(R_{\mathrm{s}}+\frac{L^{2}}{\mu C\left(V_{\mathrm{g}}-V_{\mathrm{th}}\right)}\right)^{-1}
$$

with $L=600 \mathrm{~nm}$ being the length of the channel, $\epsilon_{0}=8.854 \times$ $10^{12} \mathrm{~F} / \mathrm{m}, \epsilon_{\mathrm{HfO}_{2}}=6.5,{ }^{28}$ and $C$ the gate capacitance. The contact resistance $R_{\mathrm{s}}$, the conductance threshold voltage $V_{\mathrm{th}}$, and the mobility $\mu$ are the fit parameters. The gate capacitance was estimated numerically using a finite element simulation in COMSOL, though similar values were calculated analytically using coaxial cable or parallel plate capacitor models. The contribution of the GaAs NM to the electrical transport is negligible, as already previously demonstrated. ${ }^{27}$ 

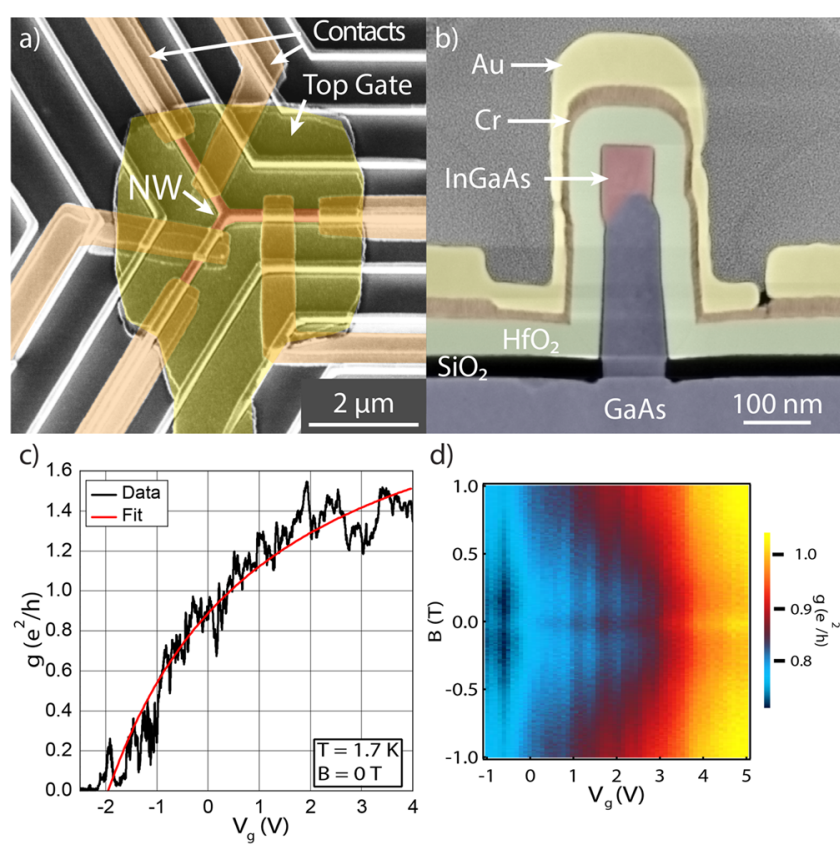

d)
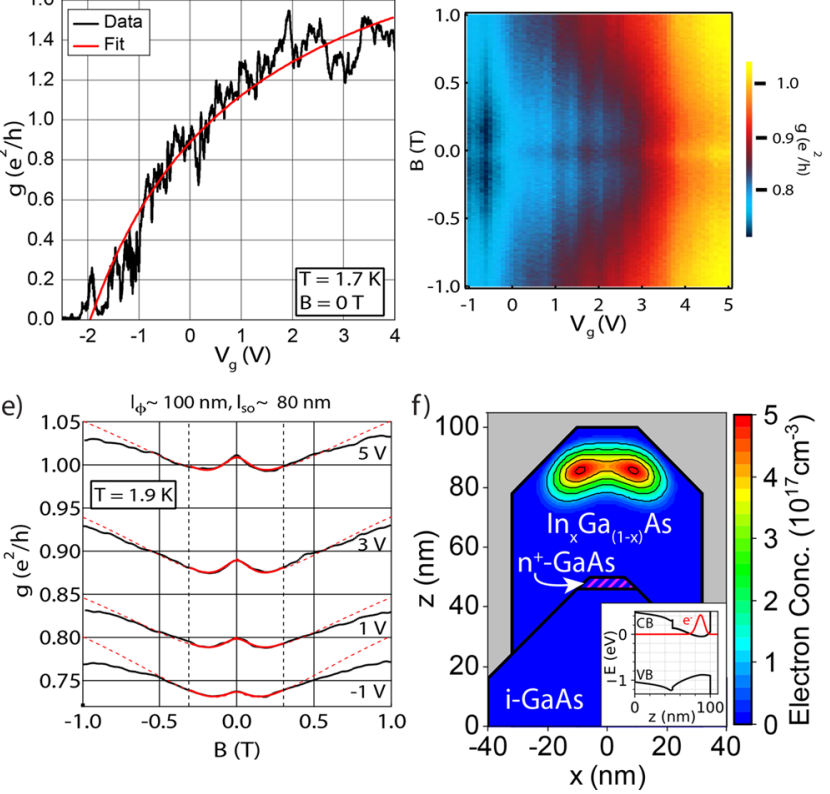

Figure 3. (a) False-colored SEM image of a fabricated electrical device showing three pairs of contacts to enable four-point measurements and a global top gate. (b) False-colored cross-sectional STEM high-angle annular dark-field (HAADF) image showing the layers in a fabricated electrical device. (c) Differential conductance as a function of top gate voltage $V_{g}$, from which the field-effect mobility $\mu=\sim 480 \pm 50 \mathrm{~cm}^{2} /(\mathrm{V} \mathrm{s})$, the conduction threshold $V_{\text {th }}=-1.9 \pm 0.2$ $\mathrm{V}$, and the contact resistance $R_{\mathrm{s}}=12.2 \pm 0.6 \mathrm{k} \Omega$ were extracted. (d) Differential conductance plot (color scale) as a function of magnetic field perpendicular to the substrate and top gate voltage with $V_{\mathrm{g}, \mathrm{AC}}=$ $200 \mathrm{mV}$ (peak-to-peak) oscillation added. (e) Magnetoconductance cuts (black) with fits (red) as labeled, exhibiting clear WAL. The WAL model is valid between the dashed vertical lines, which also denote the fitting range (solid red). Theory curves are extended to the full B-range (dashed red). Over the full gate range, average values of $l_{\phi}$ $=100 \pm 20 \mathrm{~nm}$ and $l_{\mathrm{so}}=80 \pm 20 \mathrm{~nm}$, where the mean free path $l_{\mathrm{e}}=$ $20 \mathrm{~nm}$ was held fixed here. (f) Finite element simulation in nextnano of remotely doped structure obtained from APT measurements showing an electron distribution in the InGaAs NW which is spatially separated from the n-type silicon dopants and disordered GaAs/ InGaAs interface. Inset: band structure cut along $x=0 \mathrm{~nm}$ with electron concentration overlaid in red.

We extract a field-effect mobility $\mu$ of $\sim 480 \pm 50 \mathrm{~cm}^{2} /(\mathrm{V} \mathrm{s})$. At zero gate voltage, this corresponds to a mean free path $l_{\mathrm{e}}$ of $\sim 300 \pm 30 \mathrm{~nm}$ in the $1 \mathrm{D}$ limit and $\sim 20 \pm 5 \mathrm{~nm}$ in the $3 \mathrm{D}$ limit. The associated Fermi wavelengths $\lambda_{\mathrm{F}}$ are smaller than the effective width of the nanowire, suggesting that a $3 \mathrm{D}$ approach is appropriate. Given a rather high carrier density from the capacitance models, the conductance of $\sim 1 e^{2} / h$ corresponds to partial transmission of many transverse quantum modes, corresponding to a multimode conductor. This mean free path $l_{\mathrm{e}}$ represents a significant improvement over previous work, ${ }^{27}$ where $l_{\mathrm{e}}$ was found to be limited by ionized dopant scattering on an $\sim$ nm length scale.

Reported electron mobility values in free-standing InGaAs NWs at room temperature range between 500 and $1500 \mathrm{~cm}^{2} /$ (V s), for similar In content. ${ }^{42,43}$ We associate this difference, in part, to the reduced diameter and the presence of strong surface and boundary scattering at both the top surface and disordered GaAs/InGaAs interface. In addition, the presence of alloy scattering due to disorder, as evidenced by the STEM EDS investigations, is also likely to play a role in limiting mobility in this ternary system. ${ }^{71}$ While our result is on the low end of this spectrum, we stress that our wires, grown by a SAE approach, enable scalability not achievable via standard growth techniques.

The magnetoconductance of the devices was then probed. Figure $3 \mathrm{~d}$ and e displays the conductance as a function of the applied perpendicular magnetic field $B$. Again, the results are independent of which Y-junction arms are used. The enhanced conductance at zero field indicates the presence of weak antilocalization (WAL), a hallmark signature of the SOI. WAL is the result of the accumulating spin precessions around momentum-dependent effective magnetic fields arising from spin-orbit coupling in the Dyakonov-Perel mechanism. ${ }^{72}$ In the quasiclassical, clean limit ${ }^{73-76}$

$$
\begin{aligned}
\Delta G= & \frac{e^{2}}{h} \frac{1}{L}\left[3\left(\frac{1}{l_{\phi}^{2}}+\frac{4}{3 l_{\mathrm{so}}^{2}}+\frac{1}{l_{\mathrm{B}}^{2}}\right)^{-1 / 2}-\left(\frac{1}{l_{\phi}^{2}}+\frac{1}{l_{\mathrm{B}}^{2}}\right)^{-1 / 2}\right. \\
& -3\left(\frac{1}{l_{\phi}^{2}}+\frac{1}{l_{\mathrm{e}}^{2}}+\frac{4}{3 l_{\mathrm{so}}^{2}}+\frac{1}{l_{\mathrm{B}}^{2}}\right)^{-1 / 2} \\
& \left.+\left(\frac{1}{l_{\phi}^{2}}+\frac{1}{l_{\mathrm{e}}^{2}}+\frac{1}{l_{\mathrm{B}}^{2}}\right)^{-1 / 2}\right]
\end{aligned}
$$

for a multimode quasi-1D wire, where the phase coherence length $l_{\phi}$ is exceeding the wire width $W$ and $l_{\mathrm{e}} \gg W$. Here, $e$ is the electron charge, $h$ is the Planck constant, $l_{\text {so }}$ is the spinorbit length, and $l_{\mathrm{B}}$ is the magnetic dephasing length, which in the clean limit $l_{\mathrm{e}} \gg W$ is given by

$$
l_{\mathrm{B}}{ }^{2}=\frac{C_{1} l_{\mathrm{e}} l_{\mathrm{m}}{ }^{4}}{W^{3}}+\frac{C_{2} l_{\mathrm{e}}{ }^{2} l_{\mathrm{m}}{ }^{2}}{W^{2}}
$$

where $l_{\mathrm{m}}=\sqrt{\hbar / e B}$ is the magnetic length and $C_{1,2}$ are constants dependent on the type of boundary scattering. ${ }^{73}$ Here, similar values were obtained in using both specular and diffusive boundary scattering. The coherence length, $l_{\phi}$, is a measure for how far an electron will travel on average before having its phase randomized due to inelastic scattering events. The spin-orbit length, $l_{\text {so }}$, is the typical length for the spin to precess appreciably and is, thus, a key measure for the strength of the SOI, with a shorter $l_{\text {so }}$ corresponding to stronger spinorbit coupling. We mention here that our extracted mean free path falls outside the clear validity of either of the clean $\left(l_{\mathrm{e}} \gg\right.$ $W)$ or diffusive $\left(l_{\mathrm{e}} \ll W\right)$ limits. Thus, we have done the fits in both regimes (see the Supporting Information for details), giving very good agreement with the experiment. We obtain values of $l_{\phi} \sim 100 \mathrm{~nm}$ and $l_{\mathrm{so}} \sim 80 \mathrm{~nm}$ using both the clean and diffusive limits. For the clean regime fits, $l_{\mathrm{e}}=20 \mathrm{~nm}$ was held 
fixed in order to extract $l_{\phi}$ and $l_{\text {so }}$ unambiguously. The presence of WAL indicating strong SOI in the system is also a clear advance over previous results, ${ }^{27}$ in addition to the longer mean free path. Also, the coherence and spin-orbit parameters extracted here are very similar to other studies. ${ }^{28,29,48}$

Both Dresselhaus and Rashba SOIs are key resources for topological quantum computation and spin qubits. Dresselhaus SOI, on the one hand, is due to a lack of crystal inversion symmetry. NWs, particularly those with low symmetry and/or large aspect ratio cross sections, can have a strong Dresselhaus SOI, as recently predicted. ${ }^{77}$ Rashba SOI, on the other hand, is due to a breaking of structural inversion symmetry, often appearing at an interface or surface. Both types of SOI may be gate-controlled, i.e., by changing the electric field (Rash$\mathrm{ba})^{78-80}$ or by gate-tuning the wave function asymmetry (Dresselhaus). ${ }^{2,77}$ Given the width of the present NWs, the estimated Dresselhaus contribution is negligible. We have measured the gate voltage dependence of the magnetoconductance. The resulting plot is shown in Figure $3 \mathrm{~d}$. Universal conductance fluctuations and charge switchers can often obscure the results of the magnetoconductance. In order to mitigate this, we coupled an AC-oscillation to the top gate. ${ }^{81}$ Using the model from eq 2 over the full gate voltage range, $l_{\phi}$ and $l_{\text {so }}$ are found to be essentially independent of gate voltage (see Figures S5 and S6). This can be attributed to the wraparound geometry of the gate, effectively shielding the NW from applied electric fields, while still allowing one to gate-tune the density (see Figure 3b). Thus, the WAL observed here is presumably due to the Rashba effect arising from built-in electric fields in the structure, such as gradients in the bandgap, presence of ionized dopants, or surface effects. In the Supporting Information, we give more details and a full analysis of the data with and without the AC oscillation.

\section{CONCLUSION}

We have demonstrated that remote doping of InGaAs NW networks grown by SAE is a promising approach to improving their quantum transport properties. Remote doping has enabled significant improvement of the key transport parameters such as the mean free path and SOI strength over previous bulk-doped NWs. ${ }^{27}$ Together with increased In content lowering the band gap, the Y-junctions exhibit WAL, indicating strong SOI, which is a necessary ingredient for future applications in topological quantum computation. These measurements further serve as proof of the quality of the crystal across NW Y-junctions, and this work opens up new perspectives into using this approach in networked quantum computing schemes.

\section{EXPERIMENTAL SECTION}

Growth. MBE growth was performed in a DCA P600 solidsource MBE chamber. Twenty-five nm of $\mathrm{SiO}_{2}$ was deposited on GaAs (111)B substrates by plasma-enhanced chemical vapor deposition (PEVCD). These were patterned by e-beam lithography using $35 \mathrm{~nm}$ of ZEP resist and etching in an SPTS APS dielectric etching tool employing $\mathrm{SF}_{6}$ and $\mathrm{CHF}_{3}$ chemistry. After resist stripping in an $\mathrm{O}_{2}$ plasma, the samples were etched for $10 \mathrm{~s}$ in a highly dilute buffered HF solution to remove $\sim 5 \mathrm{~nm}$ of $\mathrm{SiO}_{2}$ everywhere and smoothen the mask. Samples were then loaded and annealed at $400{ }^{\circ} \mathrm{C}$ for $2 \mathrm{~h}$ in a degassing chamber followed by $630^{\circ} \mathrm{C}$ for $30 \mathrm{~min}$ in the growth chamber immediately before growth. The GaAs buffer
NMs were grown at a temperature of $630^{\circ} \mathrm{C}$ at an equivalent 2D GaAs growth rate of $1 \AA$ As with an arsenic BEP of $4 \times 10^{-6}$ Torr. They were grown to an equivalent $2 \mathrm{D}$ thickness of 100 $\mathrm{nm}$, resulting in structures with a height of $\sim 300 \mathrm{~nm}$. Toward the end of the buffer growth, Si dopants were introduced for a short duration via a $\mathrm{Si}$ sublimation cell at a rate of $10^{13} \mathrm{~cm}^{-2}$ $\mathrm{s}^{-1}$ to achieve the desired doping profile, nominally $2 \mathrm{~nm}$ below the surface of the NM. After GaAs growth, the substrate temperature was decreased to $540{ }^{\circ} \mathrm{C}$, while the As BEP was increased to $8 \times 10^{-6}$ Torr for InAs deposition. The In flux corresponded to an InAs equivalent growth rate of $0.1 \AA / \mathrm{s}$. The In flux was closed after the $2 \mathrm{D}$ equivalent grown thickness of $60 \mathrm{~nm}$ of InAs, and the sample was then cooled down under As flux.

STEM. Cross sections of the samples were prepared first in a FEI Nova 600 Nanolab dual-beam SEM/FIB tool before being loaded into a FEI Tecnai Osiris microscope operating at 200 $\mathrm{keV}$ in STEM mode. Elemental contrast was obtained by EDS thanks to four cryo-cooled Super-X silicon drift detectors (SDDs).

APT. To protect the sample from damage during FIB milling, the sample was coated with $120 \mathrm{~nm}$ of Ni using ionbeam sputtering at $9 \mathrm{kV}$ and $7 \mathrm{~mA}$. Then, a further protective Pt capping layer was deposited by ion beam induced deposition prior to lift-out using a FEI Helios dual-beam focused ion beam. Wedge-shaped lift-outs were welded onto Si microposts, and annular milling was used to isolate the region of interest within a needle-shaped tip with a diameter of 20$60 \mathrm{~nm}$. APT analysis was performed using a local-electrode atom-probe (LEAP) 5000 XS (CAMECA, Madison, WI) with a $355 \mathrm{~nm}$ wavelength laser under the following conditions: 250 $\mathrm{kHz}$ pulse rate, $30 \mathrm{~K}$ background temperature, $2.5 \times 10^{-11}$ Torr background pressure, and $0.4 \%$ target detection rate. An initial pulse energy of $3 \mathrm{pJ}$ was used to evaporate through the surface oxides; pulses of $0.65-0.74 \mathrm{pJ}$ were used in the region analyzed. APT data were reconstructed using IVAS 3.8.5 software. The tip profile method was applied, in which SEM images of the nanotips before analysis were used to determine the reconstructed radius as a function of analyzed depth.

Contacts and Electrical Measurements. The devices were cleaned with standard solvents, and the contacts were patterned with e-beam lithography, followed by an $\mathrm{O}_{2}$ plasma cleaning. Before metallization, the samples were then exposed to an ammonium polysulfide $\left(\mathrm{NH}_{4} \mathrm{~S}_{x}\right)$ solution for $150 \mathrm{~s}$ to remove the native oxide. ${ }^{82}$ Contacts were deposited by dualangle evaporation of $14 / 50 \mathrm{~nm}$ of $\mathrm{Cr} / \mathrm{Au}$ to achieve suitable sidewall coverage. Next, $40 \mathrm{~nm}$ of $\mathrm{HfO}_{2}$ was deposited by ALD followed by another round of e-beam lithography and metallization to pattern the top gates. Finally, the sample was bonded into a chip carrier and measured using standard lock-in techniques in a variable temperature insert cryostat. Similar results were reproduced on another sample from the same wafer.

\section{ASSOCIATED CONTENT}

\section{Supporting Information}

The Supporting Information is available free of charge at https://pubs.acs.org/doi/10.1021/acs.nanolett.0c00517.

Finite element simulations, indium signal mapping by $\mathrm{X}$ ray fluorescence, further magnetotransport measurement results, and atom-probe tomography on additional heterostructures. (PDF) 


\section{AUTHOR INFORMATION}

\section{Corresponding Author}

Anna Fontcuberta i Morral - Institute of Materials, Faculty of Engineering and Institute of Physics, Faculty of Basic Sciences, Ecole Polytechnique Féderale de Lausanne, Lausanne, Switzerland; 이이. orcid.org/0000-0002-5070-2196; Email: anna.fontcuberta-morral@epfl.ch

\section{Authors}

Martin Friedl - Institute of Materials, Faculty of Engineering, École Polytechnique Féderale de Lausanne, Lausanne, Switzerland; $\odot$ orcid.org/0000-0002-7897-2550

Kris Cerveny - Department of Physics, University of Basel, Basel, Switzerland

Chunyi Huang - Department of Materials Science and Engineering, Northwestern University, Evanston, Illinois, United States

Didem Dede - Institute of Materials, Faculty of Engineering, Ecole Polytechnique Féderale de Lausanne, Lausanne, Switzerland

Mohammad Samani - Department of Physics, University of Basel, Basel, Switzerland

Megan O. Hill - Department of Materials Science and Engineering, Northwestern University, Evanston, Illinois, United

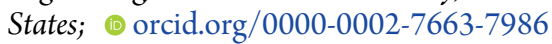

Nicholas Morgan - Institute of Materials, Faculty of Engineering, Ecole Polytechnique Féderale de Lausanne, Lausanne, Switzerland

Wonjong Kim - Institute of Materials, Faculty of Engineering, Ecole Polytechnique Féderale de Lausanne, Lausanne, Switzerland

Lucas Güniat - Institute of Materials, Faculty of Engineering, Ecole Polytechnique Féderale de Lausanne, Lausanne, Switzerland; 이이이.org/0000-0001-7883-4433

Jaime Segura-Ruiz - ESRF: the European Synchrotron, Grenoble 38043, France

Lincoln J. Lauhon - Department of Materials Science and Engineering, Northwestern University, Evanston, Illinois, United States; 10 orcid.org/0000-0001-6046-3304

Dominik M. Zumbühl - Department of Physics, University of Basel, Basel, Switzerland; 이이이.0rg/0000-0001-5831-633X

Complete contact information is available at:

https://pubs.acs.org/10.1021/acs.nanolett.0c00517

\section{Author Contributions}

${ }^{\#}$ M.F., K.C.: Equal contribution.

\section{Notes}

The authors declare no competing financial interest.

\section{ACKNOWLEDGMENTS}

The authors from EPFL and U. Basel acknowledge funding through the NCCR QSIT. Authors from EPFL further acknowledge funding from the Swiss NSF (project no. 200021_169908) and H2020 via the ITN project INDEED. Work at U. Basel was partially supported by the Swiss NSF, the Swiss Nanoscience Institute SNI, and the EU H2020 European Microkelvin Platform EMP Grant No. 824109. K.C., M.S., and D.M.Z. thank Daniel Loss and Christoph Kloeffel for useful discussions. Additional thanks to Taras Patlatiuk and Christian P. Scheller for technical support. J.S.-R. thanks the ESRF for the beamtime allocated on beamline ID16B. L.J.L. acknowledges the support of NSF DMR-1611341 and DMR-1905768.
M.O.H. acknowledges the support of the NSF GRFP. The APT sample preparation made use of the EPIC facility of Northwestern University's NUANCE Center, which has received support from the Soft and Hybrid Nanotechnology Experimental (SHyNE) Resource (NSF ECCS-1542205); the MRSEC program (NSF DMR-1121262) at the Materials Research Center; the International Institute for Nanotechnology (IIN); the Keck Foundation; and the State of Illinois through the IIN. Atom-probe tomography was performed at the Northwestern University Center for Atom-Probe Tomography (NUCAPT). The LEAP tomography at NUCAPT was purchased and upgraded with grants from the NSF-MRI (DMR-0420532) and ONR-DURIP (N00014-0400798, N00014-0610539, N00014-0910781, N00014-1712870) programs. NUCAPT received support from the MRSEC program (NSF DMR-1720139) at the Materials Research Center, the SHyNE Resource (NSF ECCS-1542205), and the Initiative for Sustainability and Energy (ISEN) at Northwestern University.

\section{REFERENCES}

(1) Garnett, E.; Mai, L.; Yang, P. Introduction: 1D Nanomaterials/ Nanowires. Chem. Rev. 2019, 119, 8955-8957.

(2) Holm, J. V.; Jørgensen, H. I.; Krogstrup, P.; Nygard, J.; Liu, H.; Aagesen, M.; Nygård, J.; Liu, H.; Aagesen, M. Surface-passivated GaAsP single-nanowire solar cells exceeding $10 \%$ efficiency grown on silicon. Nat. Commun. 2013, 4, 1498.

(3) Krogstrup, P.; Jørgensen, H. I.; Heiss, M.; Demichel, O.; Holm, J. V.; Aagesen, M.; Nygard, J.; Fontcuberta i Morral, A. Singlenanowire solar cells beyond the Shockley-Queisser limit. Nat. Photonics 2013, 7, 306-310.

(4) LaPierre, R. R.; Chia, A. C. E.; Gibson, S. J.; Haapamaki, C. M.; Boulanger, J.; Yee, R.; Kuyanov, P.; Zhang, J.; Tajik, N.; Jewell, N.; et al. III-V nanowire photovoltaics: Review of design for high efficiency. Phys. Status Solidi RRL 2013, 7, 815-830.

(5) Mallorquí, A. D.; Alarcón-Lladó, E.; Russo-Averchi, E.; Tütüncüoglu, G.; Matteini, F.; Rüffer, D.; Morral, A. F. I. Characterization and analysis of InA s/p -Si heterojunction nanowire-based solar cell. J. Phys. D: Appl. Phys. 2014, 47, 394017.

(6) Mallorquí, A. D.; Alarcón-Lladó, E.; Mundet, I. C.; Kiani, A.; Demaurex, B.; De Wolf, S.; Menzel, A.; Zacharias, M.; Fontcuberta i Morral, A. Field-effect passivation on silicon nanowire solar cells. Nano Res. 2015, 8, 673-681.

(7) Svensson, J.; Anttu, N.; Vainorius, N.; Borg, B. M.; Wernersson, L. E. Diameter-dependent photocurrent in InAsSb nanowire infrared photodetectors. Nano Lett. 2013, 13, 1380-1385.

(8) Burgess, T.; Caro, P.; Wang, Y.; Badada, B. H.; Jackson, H. E.; Smith, L. M.; Guo, Y.; Tan, H. H.; Jagadish, C.; Caroff, P.; et al. Zn3As2 Nanowires and Nanoplatelets: Highly Efficient Infrared Emission and Photodetection by an Earth Abundant Material. Nano Lett. 2015, 15, 378-385.

(9) Robson, M.; Azizur-Rahman, K. M.; Parent, D.; Wojdylo, P.; Thompson, D. A.; LaPierre, R. R. Multispectral absorptance from large-diameter InAsSb nanowire arrays in a single epitaxial growth on silicon. Nano Futures 2017, 1, 035001.

(10) LaPierre, R. R.; Robson, M.; Azizur-Rahman, K. M.; Kuyanov, P. A review of III-V nanowire infrared photodetectors and sensors. J. Phys. D: Appl. Phys. 2017, 50, 123001.

(11) Saxena, D.; Mokkapati, S.; Parkinson, P.; Jiang, N.; Gao, Q.; Tan, H. H.; Jagadish, C. Optically pumped room-temperature GaAs nanowire lasers. Nat. Photonics 2013, 7, 963-968.

(12) Mayer, B.; Rudolph, D.; Schnell, J.; Morkötter, S.; Winnerl, J.; Treu, J.; Müller, K.; Bracher, G.; Abstreiter, G.; Koblmüller, G.; et al. Lasing from individual GaAs-AlGaAs core-shell nanowires up to room temperature. Nat. Commun. 2013, 4, 2931.

(13) Tatebayashi, J.; Kako, S.; Ho, J.; Ota, Y.; Iwamoto, S.; Arakawa, $\mathrm{Y}$. Room-temperature lasing in a single nanowire with quantum dots. Nat. Photonics 2015, 9, 501-505. 
(14) Burgess, T.; Saxena, D.; Mokkapati, S.; Li, Z.; Hall, C. R.; Davis, J. A.; Wang, Y.; Smith, L. M.; Fu, L.; Caroff, P.; et al. Dopingenhanced radiative efficiency enables lasing in unpassivated GaAs nanowires. Nat. Commun. 2016, 7, 11927.

(15) Bermúdez-Ureña, E.; Tutuncuoglu, G.; Cuerda, J.; Smith, C. L. C.; Bravo-Abad, J.; Bozhevolnyi, S. I.; Fontcuberta i Morral, A.; García-Vidal, F. J.; Quidant, R. Plasmonic Waveguide-Integrated Nanowire Laser. Nano Lett. 2017, 17, 747-754.

(16) Claudon, J.; Bleuse, J.; Malik, N. S.; Bazin, M.; Jaffrennou, P.; Gregersen, N.; Sauvan, C.; Lalanne, P.; Gérard, J.-M. A highly efficient single-photon source based on a quantum dot in a photonic nanowire. Nat. Photonics 2010, 3, 116.

(17) Heiss, M.; Fontana, Y.; Gustafsson, a.; Wüst, G.; Magen, C.; O’Regan, D. D.; Luo, J. W.; Ketterer, B.; Conesa-Boj, S.; Kuhlmann, a. $\mathrm{V}$;; et al. Self-assembled quantum dots in a nanowire system for quantum photonics. Nat. Mater. 2013, 12, 439-44.

(18) Deng, J.; Su, Y.; Liu, D.; Yang, P.; Liu, B.; Liu, C. Nanowire Photoelectrochemistry. Chem. Rev. 2019, 119, 9221-9259.

(19) Jia, C.; Lin, Z.; Huang, Y.; Duan, X. Nanowire Electronics: From Nanoscale to Macroscale. Chem. Rev. 2019, 119, 9074-9135.

(20) Krogstrup, P.; Ziino, N. L. B.; Chang, W.; Albrecht, S. M.; Madsen, M. H.; Johnson, E.; Nygård, J.; Marcus, C. M.; Jespersen, T. S. Epitaxy of semiconductor-superconductor nanowires. Nat. Mater. 2015, 14, 400-406.

(21) Plissard, S. R.; van Weperen, I.; Car, D.; Verheijen, M. a.; Immink, G. W. G.; Kammhuber, J.; Cornelissen, L. J.; Szombati, D. B.; Geresdi, A.; Frolov, S. M.; et al. Formation and electronic properties of InSb nanocrosses. Nat. Nanotechnol. 2013, 8, 859-864.

(22) Car, D.; Wang, J.; Verheijen, M. A.; Bakkers, E. P. A. M.; Plissard, S. R. Rationally Designed Single-Crystalline Nanowire Networks. Adv. Mater. 2014, 26, 4875-4879.

(23) Gazibegovic, S.; Car, D.; Zhang, H.; Balk, S. C.; Logan, J. A.; de Moor, M. W. A.; Cassidy, M. C.; Schmits, R.; Xu, D.; Wang, G.; et al. Epitaxy of advanced nanowire quantum devices. Nature 2017, 548, 434-438.

(24) Kang, J.-H.; Galicka, M.; Kacman, P.; Shtrikman, H. Wurtzite/ Zinc-Blende ' $K$ '-shape InAs Nanowires with Embedded TwoDimensional Wurtzite Plates. Nano Lett. 2017, 17, 531-537.

(25) Güniat, L.; Caroff, P.; Fontcuberta i Morral, A. Vapor Phase Growth of Semiconductor Nanowires: Key Developments and Open Questions. Chem. Rev. 2019, 119, 8958-8971.

(26) McIntyre, P.; Fontcuberta i Morral, A. Semiconductor nanowires: to grow or not to grow? Materials Today Nano 2020, 9, 100058 .

(27) Friedl, M.; Cerveny, K.; Weigele, P.; Tütüncüoglu, G.; MartíSánchez, S.; Huang, C.; Patlatiuk, T.; Potts, H.; Sun, Z.; Hill, M. O.; et al. Template-Assisted Scalable Nanowire Networks. Nano Lett. 2018, 18, 2666-2671.

(28) Krizek, F.; Sestoft, J. E.; Aseev, P.; Marti-Sanchez, S.; Vaitiekenas, S.; Casparis, L.; Khan, S. A.; Liu, Y.; Stankevič, T.; Whiticar, A. M.; et al. Field effect enhancement in buffered quantum nanowire networks. Physical Review Materials 2018, 2, 093401.

(29) Vaitiekenas, S.; Whiticar, A. M.; Deng, M.-T.; Krizek, F.; Sestoft, J. E.; Palmstrøm, C. J.; Marti-Sanchez, S.; Arbiol, J.; Krogstrup, P.; Casparis, L.; et al. Selective-Area-Grown Semiconductor-Superconductor Hybrids: A Basis for Topological Networks. Phys. Rev. Lett. 2018, 121, 147701.

(30) Desplanque, L.; Bucamp, A.; Troadec, D.; Patriarche, G.; Wallart, $X$. In-plane InSb nanowires grown by selective area molecular beam epitaxy on semi-insulating substrate. Nanotechnology 2018, 29, 305705 .

(31) Aseev, P.; Fursina, A.; Boekhout, F.; Krizek, F.; Sestoft, J. E.; Borsoi, F.; Heedt, S.; Wang, G.; Binci, L.; Martí-Sánchez, S.; et al. Selectivity Map for Molecular Beam Epitaxy of Advanced III-V Quantum Nanowire Networks. Nano Lett. 2019, 19, 218-227.

(32) Lee, J. S.; Choi, S.; Pendharkar, M.; Pennachio, D. J.; Markman, B.; Seas, M.; Koelling, S.; Verheijen, M. A.; Casparis, L.; Petersson, K. D.; et al. Selective-area chemical beam epitaxy of in-plane InAs one- dimensional channels grown on $\operatorname{InP}(001), \operatorname{InP}(111) \mathrm{B}$, and $\operatorname{InP}(011)$ surfaces. Physical Review Materials 2019, 3, 084606.

(33) Karlsson, H. S.; Viselga, R.; Karlsson, U. O. Electron accumulation at the $\operatorname{InAs}(110)$ cleavage surface. Surf. Sci. 1998, 402-404, 590-594.

(34) Bryllert, T.; Wernersson, L.-E.; Froberg, L.; Samuelson, L. Vertical high-mobility wrap-gated InAs nanowire transistor. IEEE Electron Device Lett. 2006, 27, 323-325.

(35) Tanaka, T.; Tomioka, K.; Hara, S.; Motohisa, J.; Sano, E.; Fukui, T. Vertical Surrounding Gate Transistors Using Single InAs Nanowires Grown on Si Substrates. Appl. Phys. Express 2010, 3, 025003.

(36) Blömers, C.; Grap, T.; Lepsa, M. I.; Moers, J.; Trellenkamp, S.; Grützmacher, D.; Lüth, H.; Schäpers, T. Hall effect measurements on InAs nanowires. Appl. Phys. Lett. 2012, 101, 152106.

(37) Konar, A.; Mathew, J.; Nayak, K.; Bajaj, M.; Pandey, R. K.; Dhara, S.; Murali, K. V. R. M.; Deshmukh, M. M. Carrier Transport in High Mobility InAs Nanowire Junctionless Transistors. Nano Lett. 2015, 15, 1684-1690.

(38) Potts, H.; Friedl, M.; Amaduzzi, F.; Tang, K.; Tütüncüoglu, G.; Matteini, F.; Alarcon Lladó, E.; McIntyre, P. C. P.; Fontcuberta i Morral, A. From Twinning to Pure Zincblende Catalyst-Free InAs(Sb) Nanowires. Nano Lett. 2016, 16, 637-643.

(39) Joyce, H. J.; Docherty, C. J.; Gao, Q.; Tan, H. H.; Jagadish, C.; Lloyd-Hughes, J.; Herz, L. M.; Johnston, M. B. Electronic properties of GaAs, InAs and InP nanowires studied by terahertz spectroscopy. Nanotechnology 2013, 24, 214006.

(40) Boland, J. L.; Amaduzzi, F.; Sterzl, S.; Potts, H.; Herz, L. M.; Fontcuberta i Morral, A.; Johnston, M. B. High Electron Mobility and Insights into Temperature-Dependent Scattering Mechanisms in InAsSb Nanowires. Nano Lett. 2018, 18, 3703-3710.

(41) Harman, T. C.; Goering, H. L.; Beer, A. C. Electrical properties of n-type InAs. Phys. Rev. 1956, 104, 1562-1564.

(42) Hou, J. J.; Wang, F.; Han, N.; Xiu, F.; Yip, S.; Fang, M.; Lin, H.; Hung, T. F.; Ho, J. C. Stoichiometric Effect on Electrical, Optical, and Structural Properties of Composition-Tunable In x Ga 1- x As Nanowires. ACS Nano 2012, 6, 9320-9325.

(43) Hou, J. J.; Wang, F.; Han, N.; Zhu, H.; Fok, K.; Lam, W.; Yip, S.; Hung, T.; Lee, J. E.; Ho, J. C. Diameter dependence of electron mobility in InGaAs nanowires. Appl. Phys. Lett. 2013, 102, 093112.

(44) Tsui, D. C.; Stormer, H. L.; Gossard, A. C. Two-Dimensional Magnetotransport in the Extreme Quantum Limit. Phys. Rev. Lett. 1982, 48, 1559-1562.

(45) von Klitzing, K. The quantized Hall effect. Rev. Mod. Phys. 1986, 58, 519-531.

(46) Stormer, H. L. Nobel Lecture: The fractional quantum Hall effect. Rev. Mod. Phys. 1999, 71, 875-889.

(47) Shabani, J.; Kjaergaard, M.; Suominen, H. J.; Kim, Y.; Nichele, F.; Pakrouski, K.; Stankevic, T.; Lutchyn, R. M.; Krogstrup, P.; Feidenhans'L, R.; et al. Two-dimensional epitaxial superconductorsemiconductor heterostructures: A platform for topological superconducting networks. Phys. Rev. B: Condens. Matter Mater. Phys. 2016, 93, $1-6$.

(48) Kjaergaard, M.; Nichele, F.; Suominen, H. J.; Nowak, M. P.; Wimmer, M.; Akhmerov, A. R.; Folk, J. A.; Flensberg, K.; Shabani, J.; Palmstrøm, C. J.; et al. Quantized conductance doubling and hard gap in a two-dimensional semiconductor-superconductor heterostructure. Nat. Commun. 2016, 7, 12841.

(49) Nichele, F.; Drachmann, A. C.; Whiticar, A. M.; O’Farrell, E. C.; Suominen, H. J.; Fornieri, A.; Wang, T.; Gardner, G. C.; Thomas, C.; Hatke, A. T.; et al. Scaling of Majorana Zero-Bias Conductance Peaks. Phys. Rev. Lett. 2017, 119, 1-5.

(50) Suominen, H. J.; Kjaergaard, M.; Hamilton, A. R.; Shabani, J.; Palmstrøm, C. J.; Marcus, C. M.; Nichele, F. Zero-Energy Modes from Coalescing Andreev States in a Two-Dimensional Semiconductor-Superconductor Hybrid Platform. Phys. Rev. Lett. 2017, 119,176805 
(51) Gonschorek, M.; Carlin, J.-F.; Feltin, E.; Py, M. A.; Grandjean, N. High electron mobility lattice-matched AlInN/GaN field-effect transistor heterostructures. Appl. Phys. Lett. 2006, 89, 062106.

(52) Del Alamo, J. A. The high-electron mobility transistor at 30: Impressive accomplishments and exciting prospects. 2011 International Conference on Compound Semiconductor Manufacturing Technology, CS MANTECH 2011; 2011.

(53) Umansky, V.; Heiblum, M.; Levinson, Y.; Smet, J.; Nübler, J.; Dolev, M. MBE growth of ultra-low disorder 2DEG with mobility exceeding $35 \times 106 \mathrm{~cm} 2 / \mathrm{V} \mathrm{s}$. J. Cryst. Growth 2009, 311, 1658-1661.

(54) Manfra, M. J. Molecular Beam Epitaxy of Ultra-High-Quality AlGaAs/GaAs Heterostructures: Enabling Physics in Low-Dimensional Electronic Systems. Annu. Rev. Condens. Matter Phys. 2014, 5, 347-373.

(55) Boland, J. L.; Conesa-Boj, S.; Parkinson, P.; Tütüncüoglu, G.; Matteini, F.; Rüffer, D.; Casadei, A.; Amaduzzi, F.; Jabeen, F.; Davies, C. L.; et al. Modulation Doping of GaAs/AlGaAs Core-Shell Nanowires With Effective Defect Passivation and High Electron Mobility. Nano Lett. 2015, 15, 1336-1342.

(56) Boland, J. L.; Tütüncüoglu, G.; Gong, J. Q.; Conesa-Boj, S.; Davies, C. L.; Herz, L. M.; Fontcuberta i Morral, A.; Johnston, M. B. Towards higher electron mobility in modulation doped GaAs/AlGaAs core shell nanowires. Nanoscale 2017, 9, 7839-7846.

(57) Funk, S.; Royo, M.; Zardo, I.; Rudolph, D.; Morkötter, S.; Mayer, B.; Becker, J.; Bechtold, A.; Matich, S.; Döblinger, M.; et al. High mobility one- and two-dimensional electron systems in nanowire-based quantum heterostructures. Nano Lett. 2013, 13, 6189-6196.

(58) Tomioka, K.; Yoshimura, M.; Fukui, T. A., III nanowire channel on silicon for high-performance vertical transistors. Nature 2012, 488, 189-92.

(59) Leonard, D.; Krishnamurthy, M.; Reaves, C. M.; Denbaars, S. P.; Petroff, P. M. Direct formation of quantumsized dots from uniform coherent islands of InGaAs on GaAs surfaces. Appl. Phys. Lett. 1993, 63, 3203-3205.

(60) Gillin, W. P.; Homewood, K. P.; Howard, L. K.; Emeny, M. T. Thermal interdiffusion in InGaAs/GaAs strained quantum wells as a function of doping density. Superlattices Microstruct. 1991, 9, 39-42.

(61) Khreis, O. M.; Homewood, K. P.; Gillin, W. P.; Singer, K. E. Intermixing in $\mathrm{GaAsSb} / \mathrm{GaAs}$ single quantum wells. J. Appl. Phys. 1998, 84, 4017-4019.

(62) Khreis, O. M.; Homewood, K. P.; Gillin, W. P. Interdiffusion in InGaAs/GaAs: The effect of growth conditions. J. Appl. Phys. 1998, $84,232-236$.

(63) Albani, M.; Bergamaschini, R.; Salvalaglio, M.; Voigt, A.; Miglio, L.; Montalenti, F. Competition Between Kinetics and Thermodynamics During the Growth of Faceted Crystal by Phase Field Modeling. Phys. Status Solidi B 2019, 256, 1800518.

(64) Moll, N.; Kley, A.; Pehlke, E.; Scheffler, M. GaAs equilibrium crystal shape from first principles. Phys. Rev. B: Condens. Matter Mater. Phys. 1996, 54, 8844-8855.

(65) Albani, M.; Ghisalberti, L.; Bergamaschini, R.; Friedl, M.; Salvalaglio, M.; Voigt, A.; Montalenti, F.; Tütüncüoglu, G.; Fontcuberta i Morral, A.; Miglio, L. Growth kinetics and morphological analysis of homoepitaxial GaAs fins by theory and experiment. Physical Review Materials 2018, 2, 093404.

(66) Morkötter, S.; Jeon, N.; Rudolph, D.; Loitsch, B.; Spirkoska, D.; Hoffmann, E.; Döblinger, M.; Matich, S.; Finley, J. J.; Lauhon, L. J.; et al. Demonstration of Confined Electron Gas and Steep-Slope Behavior in Delta-Doped GaAs-AlGaAs Core-Shell Nanowire Transistors. Nano Lett. 2015, 15, 3295-3302.

(67) Sun, Z.; Hazut, O.; Huang, B.-C.; Chiu, Y.-P.; Chang, C.-S.; Yerushalmi, R.; Lauhon, L. J.; Seidman, D. N. Dopant Diffusion and Activation in Silicon Nanowires Fabricated by ex Situ Doping: A Correlative Study via Atom-Probe Tomography and Scanning Tunneling Spectroscopy. Nano Lett. 2016, 16, 4490-4500.

(68) Sun, Z.; Hazut, O.; Yerushalmi, R.; Lauhon, L. J.; Seidman, D. $\mathrm{N}$. Criteria and considerations for preparing atom-probe tomography specimens of nanomaterials utilizing an encapsulation methodology. Ultramicroscopy 2018, 184, 225-233.

(69) Hellman, O. C.; Vandenbroucke, J. A.; Rüsing, J.; Isheim, D.; Seidman, D. N. Analysis of Three-dimensional Atom-probe Data by the Proximity Histogram. Microsc. Microanal. 2000, 6, 437-444.

(70) Gül, Ö.; Woerkom, D. J. V.; Weperen, I. V.; Car, D.; Plissard, S. R.; Bakkers, E. P. a. M.; Kouwenhoven, L. P. Towards high mobility InSb nanowire devices. Nanotechnology 2015, 26, 215202.

(71) Harrison, J. W.; Hauser, J. R. Alloy scattering in ternary III-V compounds. Phys. Rev. B 1976, 13, 5347-5350.

(72) D’Yakonov, M. I.; Perel, V. I. Spin Orientation of Electrons Associated with the Interband Absorption of Light in Semiconductors. Sov. Phys. JETP 1971, 33, 1053.

(73) Beenakker, C. W. J.; van Houten, H. Boundary scattering and weak localization of electrons in a magnetic field. Phys. Rev. B: Condens. Matter Mater. Phys. 1988, 38, 3232-3240.

(74) Kurdak, C.; Chang, A. M.; Chin, A.; Chang, T. Y. Quantum interference effects and spin-orbit interaction in quasi-one-dimensional wires and rings. Phys. Rev. B: Condens. Matter Mater. Phys. 1992, $46,6846-6856$.

(75) Al'tshuler, B.; Aronov, A. Magnetoresistance of thin films and of wires in a longitudinal magnetic field. JETP Lett. 1981, 33, 499.

(76) van Weperen, I.; Tarasinski, B.; Eeltink, D.; Pribiag, V. S.; Plissard, S. R.; Bakkers, E. P. A. M.; Kouwenhoven, L. P.; Wimmer, M. Spin-orbit interaction in InSb nanowires. Phys. Rev. B: Condens. Matter Mater. Phys. 2015, 91, 201413.

(77) Carballido, M. J.; Kloeffel, C.; Zumbühl, D. M.; Loss, D. Lowsymmetry nanowire cross-sections for enhanced Dresselhaus spinorbit interaction. Condensed Matter2019, https://arxiv.org/abs/1910. 00562.

(78) Dettwiler, F.; Fu, J.; Mack, S.; Weigele, P. J.; Egues, J. C.; Awschalom, D. D.; Zumbühl, D. M. Stretchable Persistent Spin Helices in GaAs Quantum Wells. Phys. Rev. X 2017, 7, 031010.

(79) Hansen, A. E.; Björk, M. T.; Fasth, C.; Thelander, C.; Samuelson, L. Spin relaxation in InAs nanowires studied by tunable weak antilocalization. Phys. Rev. B: Condens. Matter Mater. Phys. 2005, 71,205328

(80) Grundler, D. Large Rashba Splitting in InAs Quantum Wells due to Electron Wave Function Penetration into the Barrier Layers. Phys. Rev. Lett. 2000, 84, 6074.

(81) Wang, R.; Deacon, R. S.; Yao, J.; Lieber, C. M.; Ishibashi, K. Electrical modulation of weak-antilocalization and spin-orbit interaction in dual gated $\mathrm{Ge} / \mathrm{Si}$ core/shell nanowires. Semicond. Sci. Technol. 2017, 32, 094002.

(82) Suyatin, D. B.; Thelander, C.; Björk, M. T.; Maximov, I.; Samuelson, L. Sulfur passivation for ohmic contact formation to InAs nanowires. Nanotechnology 2007, 18, 105307. 